\title{
On the possible mechanism of influence of change the intensity of cosmic ray on clouds anomalies at small altitudes in the Earth's atmosphere
}

\author{
I.V. Koudriavtsev ${ }^{1}$ and H. Jungner ${ }^{2}$ \\ ${ }^{1}$ Ioffe Physico-Technical Institute, Russian Academy of Sciences, Politekhnicheskaya str. 26, \\ 194021 St.Petersburg, Russia e-mail: Igor.Koudriavtsev@mail.ioffe.ru \\ ${ }^{2}$ University of Helsinki, POB 64, FIN-00014, Helsinki, Finland
}

\begin{abstract}
The possible mechanism of cosmic ray influence on clouds formation in the atmosphere is considered. Ionization of the atmosphere caused by cosmic rays can lead to the formation of condensation centers. But at the same time interaction between cosmic ray and the atmosphere might change transparency of the atmosphere and the atmospheric temperature, too. Calculations of changes of the condensation growth rate of water drops due to changes of the temperature have been performed. This effect can provide an increase in a few percents of growth rate of drops. This can explain the observed correlation between cloudiness and cosmic ray intensity.
\end{abstract}

\section{Introduction}

The cosmic ray influence on climatic changes is widely discussed today (for example Van Geel et al. 1999; Dergachev et al. 2000). One issue under consideration is the possible connection between variations in the cosmic ray fluxes and cloud formation. Marsh \& Svensmark (2000) have shown that a correlation between cloudiness at heights up to 3.2 $\mathrm{km}$ and the intensity of galactic cosmic rays (GCR) exists. This fact indicates that GCR can affect the formation of clouds in the atmosphere. It is supposed, that ionization of the atmosphere by GCR results in the formation of aerosols, which serve as additional nuclei of the condensation of the drops. However, mechanisms of these processes are not clear. Generation of additional aerosols and multi-atomic molecules will cause not only change in the amount of condensation nuclei but also change optical parameters of the atmosphere as they can scatter and absorb radiation. It will result in change of its thermodynamic parameters and impact on clouds formation. Here we consider the mechanism of GCR influence on changes of thermodynamic parameters of the atmosphere and consequently on clouds formation. It is known, that transparency of the atmosphere for visible radiation decreases by $10 \%$ and the temperature of the lower atmosphere increases after the beginning of solar proton events (Pudovkin \& Morozova, 1997). The assumption is that during periods of solar proton events a layer with reduced transparency is formed in the atmosphere at the height of $8-10 \mathrm{~km}$. This layer may absorb or reflect solar radiation. This layer can consist of multiatomic molecules, aerosols and ions formed as a result of ionizing actions of CR. For an explanation of the temperature change during solar proton events Pudovkin \& Morozova (1997) assumed that this layer can reflect up to $10 \%$ of visible radiation and up to $40 \%$ of infrared radiation. Due to this temperature in the atmosphere can change by $2-3 K$. It is logical to assume, that the layer changing transparency of the atmosphere can be formed not only under action of solar protons but by GCR protons also. 


\section{The model and the results of calculations}

So, we consider that protons of CR generate a layer reducing atmospheric transparency and that the variation in transparency is anticorrelated with the variation of the CR intensity. We consider absorption in the layer because it is known that multiatomic molecules $\left(\mathrm{CO}_{2}, \mathrm{H}_{2} \mathrm{O}\right.$ and other $)$ absorb radiation effectively. For determination of temperature we use the same method as in Hrgian (1978). Let $W$ is the flux density of solar visible radiation in the atmosphere which take into account the albedo effect; $A$ and $B$ are is flux density of the infra-red radiation going downwards and upwards. The axis $Z$ is directed upwards. The equations for these fluxes can be written as follows. Part of the solar radiation, which is going downwards is absorbed in the layer with a thickness dz,

$$
d W=\alpha_{1}^{\prime} \rho W \sec \xi d z+\alpha^{\sim} \rho^{\sim} W \sec \xi d z=\alpha_{1} \rho\left(1+\delta_{1}\right) W d z
$$

where $\alpha_{1}=\alpha_{1}^{\prime} \sec \xi ; \delta_{1}=\alpha^{\sim} \rho \sim / \alpha_{1}^{\prime} \rho ; \alpha_{1}^{\prime}$ - mean factor of absorption of the visible radiation; $\xi$-the angle of arrival of the solar radiation; $\rho$ - density of the principal absorption matter ; $\alpha^{\sim}$ - additional factor of absorption of the visible radiation due to the impact of $\mathrm{CR}$ on the atmosphere; $\rho^{\sim}$ - density of the additional absorption matter. Then, the equation for flux of the visible radiation is

$$
\frac{d W}{d z}=\alpha_{1} \rho\left(1+\delta_{1}\right) W
$$

Similarly, according to Hrgian (1978) for flux of the infrared radiation we have:

$$
\frac{d A}{d z}=\alpha_{2} \rho\left(1+\delta_{2}\right)(A-f E) ; \frac{d B}{d z}=\alpha_{2} \rho\left(1+\delta_{2}\right)(f E-B)
$$

where $\alpha_{2}$ - average factor of absorption of the infra-red radiation in the atmosphere, $\delta_{2}$ describes additional absorption of the infra-red radiation due to the influence of $\mathrm{CR}$ on the atmosphere, $E=\sigma T^{4}, \sigma$ - the Stefan - Boltzman constant , $T$ - the air temperature. Factor $f$ describes the deviation of air radiation from the "black body" radiation. The equation of heat input $d Q$ to the layer $d z$ is (according to Hrgian 1978).

$$
\frac{d Q}{d z}=\alpha_{1} \rho\left(1+\delta_{1}\right) W+\alpha_{2} \rho\left(1+\delta_{2}\right) A+\alpha_{2} \rho\left(1+\delta_{2}\right) B-2 \alpha_{2} \rho\left(1+\delta_{2}\right) f E+\frac{d}{d z} \lambda \frac{d T}{d z}
$$

where $\lambda$ describes turbulent heat conductivity. This equations differ from the equations of Hrgian (1978) by presence of functions $\delta_{1}$ and $\delta_{2}$. We have solved the equations (2.22.4) for various values of additional absorption of radiation in the visible and infra-red ranges, i.e. for different $\delta_{1}$ and $\delta_{2}$ neglecting the heat conductivity(i.e. $\lambda=0$, according to Hrgian (1978)) by presence of functions $\delta_{1}$ and $\delta_{2}$. The solution for $T$ in the case of absence of an additional absorbing layer $\left(\delta_{1}=0, \delta_{2}=0\right)$ will be

$$
\sigma T_{1}^{4}=\frac{W_{0}}{2 f}\left(1+\frac{1}{\beta}-\left(\frac{1}{\beta}-\beta\right) e^{-\beta \tau}\right) ; \beta=\alpha_{1} / \alpha_{2}
$$

i.e. the solution becomes similar to that of Hrgian (1978), where $\beta=0,2$. Results of calculations of the temperature $T\left(\Delta T=T-T_{1}\right)$ are shown on figures 1 for various values of additional absorption of radiation in the visible and infra-red ranges. We used the following functions for $\delta_{1}$ and $\delta_{2}$ :

$$
\delta_{1}=\frac{\delta_{10}}{\sqrt{2 \pi} \sigma_{1}} \exp \left(-\left(\tau_{v}-\tau_{v 0}\right)^{2} /\left(2 \sigma_{1}^{2}\right)\right) ; \delta_{2}=\frac{\delta_{20}}{\sqrt{2 \pi} \sigma_{2}} \exp \left(-\left(\tau-\tau_{0}\right)^{2} /\left(2 \sigma_{2}^{2}\right)\right)
$$

where $\sigma_{1}=\beta \sigma_{2} ; \tau_{v 0}=\beta \tau_{0} ; \tau_{v}=\int_{z}^{\infty} \alpha_{1} \rho(h) d h$, i.e. $\tau_{v}=\beta \tau$.

As we can see from the figures 1 , presence of a layer with additional absorption leads to the increase of temperature in the lower atmosphere by $1-2 K$. 


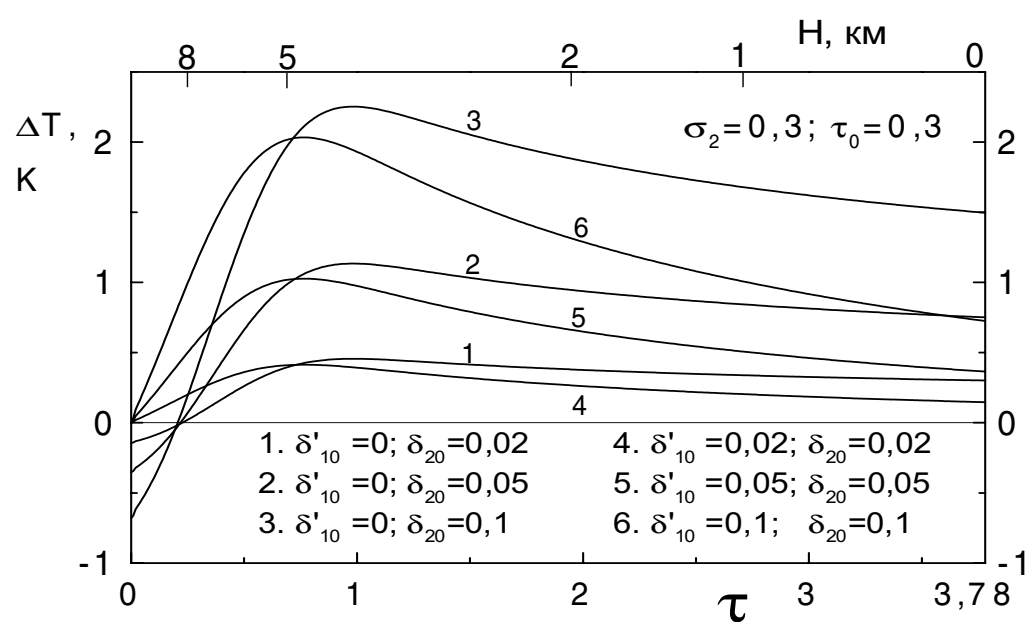

Figure 1. Change of temperature for different values of $\tau$ (for various heights $Z$ ). Conversion from $\tau$ to $Z$ is according to Hrgian (1978). $\delta_{10}^{\prime}=\delta_{10} / \beta$

The rate of condensation growth of droplets can be described by modified Maxwell formulae which would take into account the heat of condensation and forces of the surface tension (Sedunov 1972)

$$
\frac{d R}{d t}=\frac{D \rho}{R \rho_{1}}\left(\delta_{0}-\frac{2 \sigma_{t}}{\rho_{1} R_{v} T R}\right) /\left(1+\frac{\rho L D}{k_{1} T}\left(\frac{L}{R_{v} T}-1\right)\right)
$$

where $R$ - the radius of drop, $\sigma_{t}$ - the coefficient of surface tension, $D$ - the coefficient of vapor diffusion, $\rho$ - the density of vapor, $\rho_{1}$ - the density of water, $\delta_{0}$ - the coefficient of vapor supersaturation, $R_{v}$ - gas constant of vapor, $L$ - the heat of condensation, $k_{1}$ - the coefficient of air thermal conductivity. The parameters $D, \sigma_{t}, L, k_{1}$ are functions of the thermodynamical parameters of the atmosphere (Sedunov 1972). This equation describes growth of drops in the atmosphere initially heated up to the temperature $T$ during slow cooling, when the following condition is satisfied $\delta_{0}=1-\rho_{0}(T) / \rho<<1$, i.e. for $\rho \approx$ $\rho_{0}(T), \rho_{0}$ - the density of saturated vapor. To express $\rho$ from $\delta_{0}$ and $\rho_{0}, \rho=\rho_{0}(T) /\left(1-\delta_{0}\right)$. The results of calculations made using the eq.(2.7) for the stable pressure are shown on the figure 2. The changes of the drop growth rate are shown for the part of bottom atmosphere where the temperature $t>-5^{\circ} C$. The existence of liquid phase is possible at such altitudes, moreover as a condensation nuclei there might be a drop of saturated solution of $\mathrm{NaCl}$ (Aleksandrov et al. 1967). As we can see, the presence of a layer with additional absorption leads to the increase of temperature of the lower atmosphere and results in increase of the growth rate of droplets under this layer. The increase of rate of the droplets growth in the atmosphere may reach a few percents. It should be mentioned that the calculations have been performed in the present work were made for the fixed (though different) values of the $\delta_{0}$ parameter. More detailed consideration of the present problem requires solving a self-consistent task, which would take into account temporary changes of the $\delta_{0}$ parameter at various altitudes. However, the present investigation shows that changes of the atmospheric transparency might lead to changes of condensed growth of atmospheric droplets by few percents and, hence, result in cloudiness variations. 


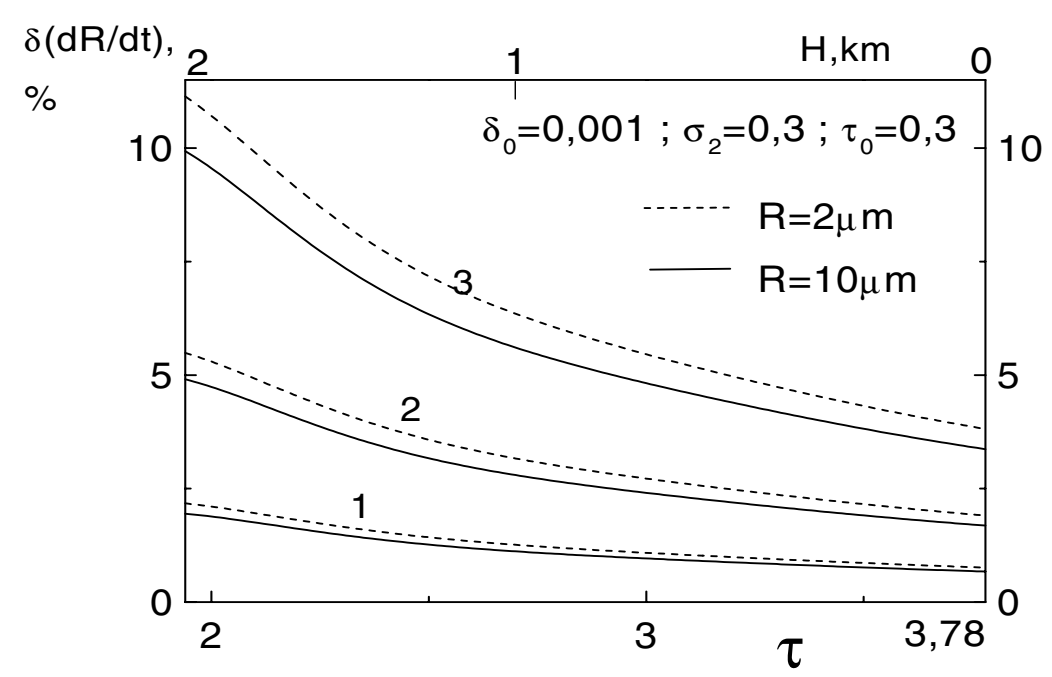

Figure 2. Change of the rate of drop growth for various heights: 1$) \delta_{10}^{\prime}=0,02 ; \delta_{20}=0,02$;

$$
\begin{gathered}
\left.2) \delta_{10}^{\prime}=0,05 ; \delta_{20}=0,05 ; 3\right) \delta_{10}^{\prime}=0,1 ; \delta_{20}=0,1 \\
\delta(d R / d t)=\frac{(d R / d t)_{T(z)}-(d R / d t)_{T_{1}(z)}}{(d R / d t)_{T_{1}}(z)} \times 100 \%
\end{gathered}
$$

\section{Conclusion}

In conclusion we note that the detailed model describing cosmic ray influence on clouds formation should take into account change of the transparency of the Earth's atmosphere and its thermodynamic parameters. Change of the atmospheric transparency caused by cosmic rays can change temperature of the atmosphere and affect growth rate of drops. The increase of growth rate of the droplets may reach a few percents and therefore results in increase of clouds cover. This can explain the observed correlation between cloudiness and cosmic rays.

\section{Acknowledgements}

This research was done in the frame of exchange between the Russian and Finnish Academies (project $\mathrm{u}$ 16) and was supported INTAS grant 2001-0550 and grants RFBR 03-02-17505, 04-02-17560. Authors are thankful to Dr. Y. Kartavykh and Dr. M. Ogurtsov for their useful advices and aid.

\section{References}

Aleksandrov E.L., Levin L.M. \& Sedunov Y.S. 1967 Izvestiy Academy of Sciences, Physics of the atmosphere and the ocean. 3, 872-880, (in Russian).

Dergachev V.A., Raspopov O.M., Shumilov O.I., Van Geel B., Morner N.-A., Van der Plicht J. \& Rensen H. 2000 In Proc 1st Solar \& Space Weather Euroconference "The Solar Cycle and Terrestrial Climate", Santa Cruz de Tenerife, Spain, 25-29 September 2000, 513-516.

Hrgian A.H. 1978 Physics of atmosphere Leningrad (in Russian).p360

Marsh N. \& Svensmark H. 2000 Space Science Reviews 94, 215-230.

Pudovkin M.I. \& Morozova A.L. 1997 Journal of Atmospheric and Solar - Terrestrial Physics 59, 2159-2166.

Sedunov Y.S. 1972 The Physics of generation of the liquid phase in the atmosphere , Leningrad, p208 (in Russian).

Van Geel B., Raspopov O.M., Dergachav V.A., Renseen H. \& Van der Plicht J. 1999 Quaternary Science Reviews 18, 331-338. 